\title{
First Calculation of Nuclear Parity Violation from Lattice QCD
}

\section{Joseph Wasem*}

Lawrence Livermore National Laboratory, L-414, 7000 East Ave., Livermore, CA 94550, USA

E-mail: wasem2dIInL.gov

LLNL-JRNL-491671

We present the first lattice QCD calculation of the leading-order momentum-independent parity violating coupling between pions and nucleons, $h_{\pi N N}^{1}$. The calculation performs measurements on a single ensemble of dynamical anisotropic clover gauge configurations, with a spatial extent of $L \sim 2.5 \mathrm{fm}$, a spatial lattice spacing of $a_{s} \sim 0.123 \mathrm{fm}$, and a pion mass of $m_{\pi} \sim 389 \mathrm{MeV}$. We find a contribution from the 'connected' diagrams of $h_{\pi N N}^{1, c o n}=\left(1.099 \pm 0.505_{-0.064}^{+0.058}\right) \times 10^{-7}$, consistent with current experimental bounds and previous model-dependent theoretical predictions.

XXIX International Symposium on Lattice Field Theory

July 10 - 162011

Squaw Valley, Lake Tahoe, California

${ }^{*}$ Speaker. 
The combination of QCD and electroweak interactions underlies all of nuclear physics, and quantitative explorations of nuclear observables directly from QCD using lattice QCD in the nonperturbative regime are becoming accessible. This is particularly meaningful for processes which are poorly understood experimentally, such as the neutral current parity violating (PV) weak interaction between quarks. Indeed, this interaction stands apart as the least understood portion of the standard model. Here we report on the first calculation directly from QCD of the leadingorder momentum-independent parity violating coupling between pions and nucleons, $h_{\pi N N}^{1}$, using $n_{f}=2+1$ lattice QCD calculations on configurations with a pion mass of $m_{\pi} \sim 389 \mathrm{MeV}$.

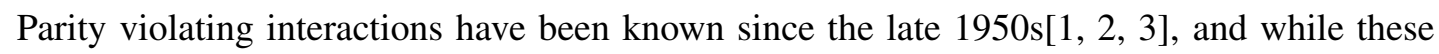
interactions can be studied in flavor-changing decays the effects of the PV neutral-current in such decays are tiny, as the tree-level coupling between quarks and the $Z$ boson are flavor diagonal and

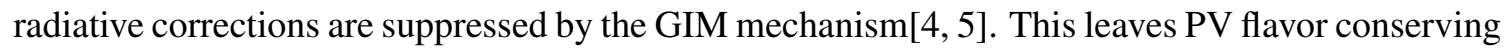
interactions as the only laboratories for studying the weak neutral current, with the nucleon-nucleon (NN) PV interaction as the only accessible case. Isolation of the hadronic weak neutral current occurs in the $\Delta I=1 \mathrm{NN}$ channel, and this component is thought to be dominated by long-range pion exchange[团, 目].

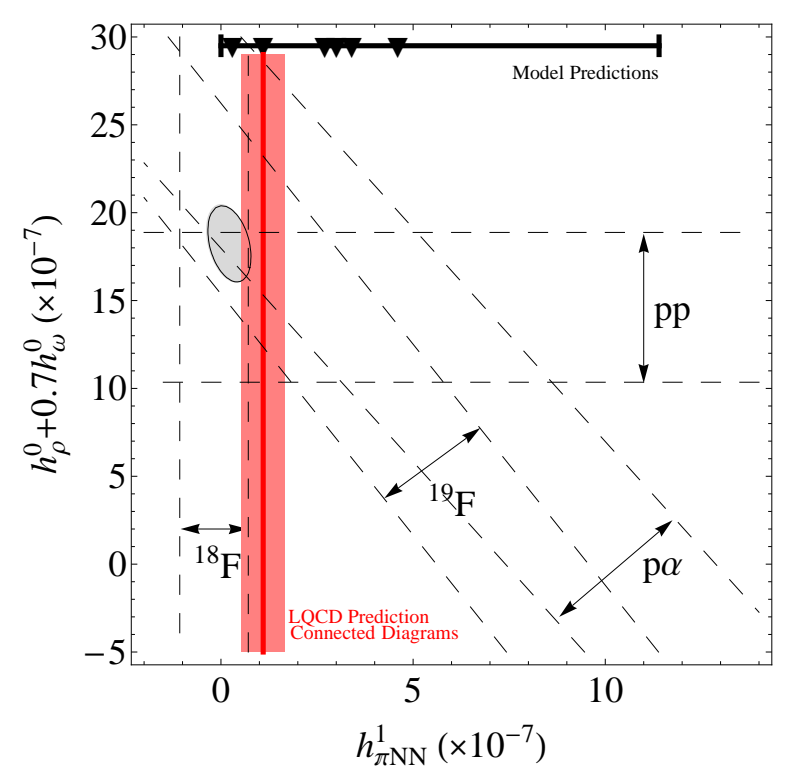

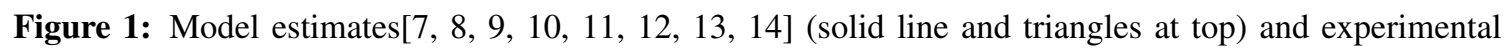
results (dashed lines with labels and $1 \sigma$ error ellipse in grey, from Refs. [四, [5], [6] and references therein) for $h_{\pi N N}^{1}$ versus the dominant isoscalar PV coupling combination, along with the results of this work (solid vertical line and error band).

At hadronic scales the weak interaction can be qualitatively considered as a pointlike fourquark interaction which gives rise to a pion that mediates long-range interactions. Experiments to uncover this effect are technically demanding however, as the ratio of the weak to strong contributions to the $\mathrm{NN}$ interaction is approximately $10^{-7}$. In the decades since the discovery of parity violation, a heroic series of experiments (see Refs. [四, [5, [6] and references therein) have sought 
to uncover the value of $h_{\pi N N}^{1}$, defined as

$$
\mathscr{L}_{P V}^{\pi N N}=h_{\pi N N}^{1}\left(\bar{p} \pi^{+} n-\bar{n} \pi^{-} p\right)
$$

with a proton $(p)$, neutron $(n)$, and pion $(\pi)$. The most precise of these experiments are plotted with dashed lines in Fig. 四, with the combined $1 \sigma$ error ellipse shown in grey. The coupling $h_{\pi N N}^{1}$ dominates the long range parity violating NN potential as it is not suppressed by powers of momentum. Although lacking precision, experimental results thus far suggest that while the isoscalar $\mathrm{PV}$ interaction is of natural size, the isovector interaction $h_{\pi N N}^{1}$ is suppressed. Early results from the most recent experimental collaboration to examine nuclear parity violation, the NPDGamma collaboration[[]], have thus far not provided any significant constraint on $h_{\pi N N}^{1}$. However, the experiment is currently being reinstalled at the Spallation Neutron Source at Oak Ridge National Laboratory and should very soon be able to reach its design precision.

Several model-dependent attempts have been made to calculate $h_{\pi N N}^{1}$ in such a way that the nonperturbative QCD effects are included. The earliest of these used the quark model in combination with several symmetry considerations to make the first robust theoretical predictions of $h_{\pi N N}^{1}[$ [U4] (the DDH result). Despite a tremendous amount of effort, the remaining systematic uncertainties from the nonperturbative sector of QCD prevented Ref. [14]] from specifying a result, and instead the outcome of the calculation was presented as a 'best guess' and an accompanying

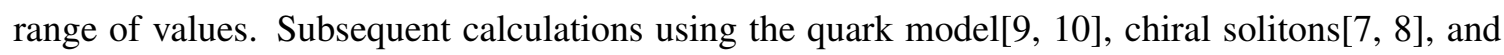
QCD sum rules[Ш], 미, [3] have obtained greatly varying values of $h_{\pi N N}^{1}$, but all have remained within the original DDH range. The DDH range and the results of each model calculation are shown at the top of Fig. $\mathbb{\text { W. }}$

The calculation presented here uses one ensemble of $n_{f}=2+1$ anisotropic clover gauge configurations with a pion mass of $m_{\pi} \sim 389 \mathrm{MeV}$, spatial lattice spacing of $a_{s} \sim 0.123(1) \mathrm{fm}$, and an anisotropy of $\xi=3.50(3)\left[\mathbb{8}\right.$, [प] ]. The configurations have a total extent of $20^{3} \times 256$, leading to a spatial dimension of $L \sim 2.5 \mathrm{fm}$ and $m_{\pi} L \sim 4.9$. Three-point correlation functions of the form

$$
C_{A \rightarrow B}^{i j}\left(t, t^{\prime}\right)=\left\langle 0\left|\mathscr{O}_{B, j}(t) \mathscr{O}_{P V}^{\Delta I=1}\left(t^{\prime}\right) \mathscr{O}_{A, i}^{\dagger}(0)\right| 0\right\rangle
$$

are constructed, with $t$ the sink timeslice and $t^{\prime}$ the operator insertion timeslice. $\mathscr{O}_{n, k}$ is an interpolating operator with the quantum numbers of state $n$ and source/sink smearing of type $k$. Sandwiched between these operators is the four-quark operator for the $\Delta I=1 \mathrm{PV}$ interaction. Two-point correlation functions of the form $C_{n}^{i j}(t)=\left\langle 0\left|\mathscr{O}_{n, j}(t) \mathscr{O}_{n, i}^{\dagger}(0)\right| 0\right\rangle$ are also calculated. The interpolating operator used for the proton is $\varepsilon^{a b c} u_{a}^{\alpha}\left(d_{b}^{\beta}\left[C \gamma_{5}\right]_{\beta \delta} u_{c}^{\delta}\right)$, with color indices $a, b, c$ and spin indices $\alpha$, $\beta, \delta$. A similar operator, $\varepsilon^{a b c}\left[\gamma_{5}\right]_{\alpha \omega} u_{a}^{\omega}\left(d_{b}^{\beta}\left[C \gamma_{5}\right]_{\beta \delta} u_{c}^{\delta}\right)$, creates a state with the quantum numbers of the neutron-pion in an S-wave [20, 2], [22, [23] (expected to be the $n \pi$ system rather than the $S_{11}$ negative parity proton at this pion mass). Using a three-quark interpolating operator to create the $n \pi$ state greatly simplifies the quark contractions necessary for the 3-point function. Furthermore, the simplified operator removes the need to calculate expensive quark-loop contributions at the sink, which would arise from separate $n$ and $\pi$ interpolating operators.

The four-quark $\Delta I=1 \mathrm{PV}$ operator can be constructed directly from the standard electroweak interaction Lagrangian[[] at the scale of the weak gauge bosons by integrating out the $Z$ boson (the contributions from the exchange of the $W^{ \pm}$bosons are neglected as they are suppressed by 
$\sin ^{2}\left(\theta_{C}\right) \approx 0.05$, where $\theta_{C}$ is the Cabibbo angle). One can then use continuum one-loop QCD perturbation theory to run the operator coefficients to the scale of the hadronic interactions $\left(\Lambda_{\chi}=1\right.$ $\mathrm{GeV}$ ) integrating out the heavier $b$ - and $c$-quarks along the way[24, 目]. During the course of this running mixing between operators with the same quantum numbers will occur, leaving a total of 8 operators at the hadronic scale. There is no mixing with lower-dimension operators as the $\Delta I=1 \mathrm{PV}$ operator also conserves $\mathrm{CP}$, precluding quark bilinear operators from contributing with divergent powers of the lattice spacing. The full four-quark $\Delta I=1 \mathrm{PV}$ operator at the hadronic scale can then be expressed as

$$
\mathscr{O}_{P V}^{\Delta I=1}=-\frac{G_{F} \sin ^{2}\left(\theta_{W}\right)}{3 \sqrt{2}} \sum_{i=1}^{4} \int d^{3} x\left(C_{i} \theta_{i}^{q}+S_{i} \theta_{i}^{s}\right)
$$

where $G_{F}=1.16637 \times 10^{-5} \mathrm{GeV}^{-2}$ is the Fermi coupling and $\sin ^{2}\left(\theta_{W}\right)=0.231$ is the weak mixing angle[25]. The four-quark operators that contain only light ( $u$ and $d$ ) quarks are $\theta_{i}^{q}$, while the $\theta_{i}^{s}$ contain $s$-quarks along with light quarks. The coefficients $C_{i}$ and $S_{i}$ of these operators and the specific operator forms used for $\theta_{i}$ in this work can be found in Ref. [26].

Performing the quark contractions in the three-point correlation function of Eq. $\square$ using the above operators, one arrives at three possible diagrams for the quark propagators. The first type connects two of the quarks from both the source and sink operators to the weak operator, with the third quark going directly between the source and sink. This type is drawn in Fig. $\mathbb{Z}$ (a) and is called the 'connected' case. The second, 'quark-loop' type of Fig. $D(b)$ contains a quark loop at the weak operator insertion while connecting only one quark each from the source and sink to the weak operator. The final type contains a weak operator where all four quarks are contracted with each other, leading to an entirely 'disconnected' contribution. However, in the isospin limit the contributions from this type of diagram will sum to zero, saving considerable computational expense. Because the interpolating operators consist entirely of light quarks, the operators $\theta_{i}^{q}$ will have contributions to both the connected and quark-loop diagrams, while the operators $\theta_{i}^{s}$ will contribute only to the quark-loop diagrams as the $s$-quarks will be required to be contained in the quark loop itself.

(a)

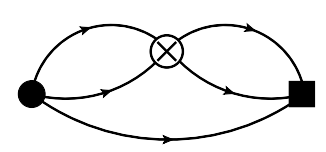

(b)

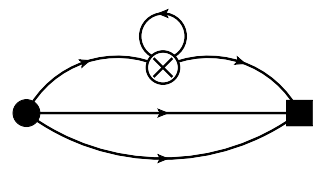

Figure 2: The (a) connected and (b) quark-loop diagrams that contract the parity-violating operator with the interpolating operators for the source and sink. The filled circle and square represent the three-quark interpolating operators used at the source and the sink respectively, with one positive party and the other negative parity.

The technique of sequential propagators fails for this calculation both in the case of the connected diagrams (due to the need for two propagators between the operator and the sink) and in the case of the quark loop diagrams (as the quark-loop would remain to be calculated). Instead, this calculation performs two separate quark propagator inversions, one at the source and one at the weak operator insertion. This method unfortunately restricts the measurements to a single spatial site on the operator timeslice (all spatial sites are sampled over the course of the calculation), 
but allows for maximum flexibility and computational efficiency (as the propagators may be used for both the connected and the quark-loop diagrams, and for any of the weak operators). With this method, the timeslice on which the weak operator is placed $\left(t^{\prime}\right)$ must be large enough that the excited states of the source operator are exponentially small, and for this calculation $t^{\prime}=24$.

To extract the desired matrix element, a ratio of 3-point and 2-point functions must be formed such that in the limit of large $t^{\prime}$ and $t-t^{\prime}$ contamination from excited states dies off and the ground state overlap factors are canceled, allowing the ratio to plateau to the value of the matrix element. This ratio is given by

$$
R_{A \rightarrow B}^{i j}=\frac{C_{A \rightarrow B}^{i j}\left(t, t^{\prime}\right)}{C_{B}^{j j}(t)}\left(\frac{C_{A}^{i i}\left(t-t^{\prime}\right) C_{B}^{j j}(t) C_{B}^{j j}\left(t^{\prime}\right)}{C_{B}^{j j}\left(t-t^{\prime}\right) C_{A}^{i i}(t) C_{A}^{i i}\left(t^{\prime}\right)}\right)^{\frac{1}{2}}
$$

where the smearing used in the 2-point functions must match that used for the corresponding state in the 3-point function in order to have the correct cancelation of overlap factors. However, as discussed in Ref. [26] the differing energy levels of the proton and the $n \pi$ states will cause an insertion of energy by the weak operator to occur, modifying Eq. 1 to

$$
\mathscr{L}_{P V}^{\pi N N}=h_{\pi N N}^{1}\left(\bar{p} \pi^{+} n-\bar{n} \pi^{-} p\right)+h_{E} D_{t}\left(\bar{p} \pi^{+} n-\bar{n} \pi^{-} p\right)
$$

with some unknown coefficient $h_{E}$, making the long-time $\left(\left(t-t^{\prime}\right) \rightarrow \infty\right)$ behavior of Eq. 田

$$
R_{p \rightarrow n \pi}^{i j} \rightarrow h_{\pi N N}^{1}+\Delta E \cdot h_{E}
$$

To remove the $\Delta E$ dependence one observes that the first term in Eq. 5 is antisymmetric with respect to the interchange of the proton and $n \pi$ initial and final states, while the second term is symmetric. Thus the energy injection term can be eliminated with an antisymmetric combination of Eq. leading to a plateau region given by

$$
H^{i j}=\frac{1}{2}\left(R_{p \rightarrow n \pi}^{i j}-R_{n \pi \rightarrow p}^{i j}\right) \rightarrow h_{\pi N N}^{1}
$$

A total of 100,871 measurements of each of the smearing combinations of $H^{i j}$ are performed, where $i, j$ can be either point- or shell-smearing. These measurements are then blocked on each configuration and bootstrapped. One can enhance the plateau region for Eq. $\square$ by taking appropriately normalized linear combinations of the different smearing combinations, using the matrix-prony [27] method on the bootstrapped ensemble to determine the optimal linear combination. This is done for both the connected and the quark-loop contractions. In the case of the quark-loop diagrams the signal-to-noise ratio remains far too small to recover any reliable result, and indeed it is not possible to even define a plateau region. It is expected that improvements in both contraction algorithms and overall calculation runtime will be needed to overcome this difficulty and reliably extract the quark-loop contribution. For the connected contributions the analysis returns the data shown in Fig. [1, revealing not only a well defined plateau region, but a robust non-zero contribution to $h_{\pi N N}^{1}$.

In Fig. [1], a fully correlated $\chi^{2}$ minimizing fit to a constant is performed over the plateau region, with additional systematic error due to the choice of plateau region determined by shifting the ends of the region \pm 2 timeslices. The quoted systematic error is then one-half of the maximum minus the minimum of these shifted fits. The fit result and statistical plus systematic error are shown in 


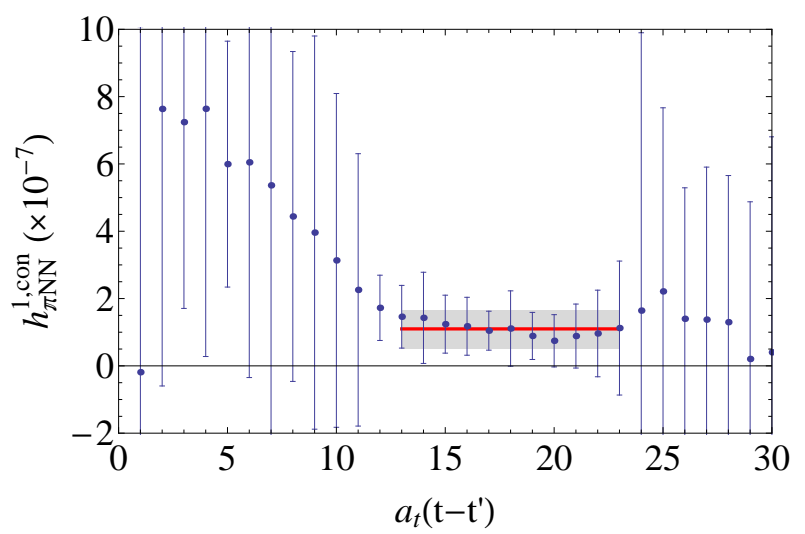

Figure 3: Lattice results for the contribution of connected quark diagrams to $h_{\pi N N}^{1}$, as a function of Euclidean lattice time from the operator insertion. The solid line is the fully correlated fit value over the plateau region with the grey rectangle the statistical plus fit window systematic uncertainty.

Fig. [] with the solid line and grey band. The contribution of the connected diagrams to $h_{\pi N N}^{1}$ is then found to be

$$
h_{\pi N N}^{1, c o n}=\left(1.099 \pm 0.505_{-0.064}^{+0.058}\right) \times 10^{-7}
$$

where the first and second uncertainties are statistical and systematic, respectively. Comparing the error bars on the points with the error band from the fit, one observes a high degree of correlation between successive timeslices, which results from the lattice anisotropy. The fit result is plotted in

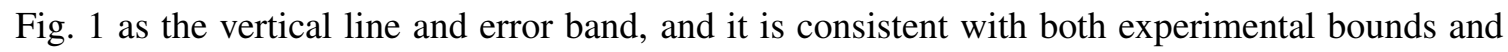
previous model calculations.

In conclusion, we have performed the first calculation of the quantity $h_{\pi N N}^{1}$ directly from the underlying theory of QCD. Our calculation was performed on one ensemble of anisotropic clover configurations with a pion mass of $m_{\pi} \sim 389 \mathrm{MeV}$. Future calculations will need to be performed at pion masses closer to the physical point, and with sufficient statistical resolution to extract the contribution of the quark-loop diagrams (expected to be on the order of $10^{3}$ more measurements). Our result, while incomplete, shows good agreement with current experimental bounds and paves the way toward a complete extraction of $h_{\pi N N}^{1}$ at a precision consistent with, or better than, the anticipated results of the upcoming NPDGamma experiment at Oak Ridge.

We thank M. J. Savage, T. Luu, A. Nicholson, B. Smigielski, A. Walker-Loud, B. Tiburzi, M. Buchoff, W. Haxton and P. Vranas for many useful discussions and B. Joo for help with QDP++ and CHROMA[[28]. The configurations used were generated on $\mathrm{UBGL}$ while the propagator inversions and contractions were performed on the Edge cluster, both at LLNL. This work was performed under the auspices of the U.S. Department of Energy by LLNL under Contract No. DE-AC5207NA27344 and the UNEDF SciDAC Grant No. DE-FC02-07ER41457. Preliminary work at the University of Washington was performed under DOE Grant No. DE-FG02-97ER41014.

\section{References}

[1] T. D. Lee, C. -N. Yang, Phys. Rev. 104, 254-258 (1956).

[2] C. S. Wu, E. Ambler, R. W. Hayward, D. D. Hoppes, R. P. Hudson, Phys. Rev. 105, 1413-1414 (1957). 
[3] R. L. Garwin, L. M. Lederman, M. Weinrich, Phys. Rev. 105, 1415-1417 (1957).

[4] E. G. Adelberger, W. C. Haxton, Ann. Rev. Nucl. Part. Sci. 35, 501-558 (1985).

[5] S. L. Glashow, J. Iliopoulos, L. Maiani, Phys. Rev. D2, 1285-1292 (1970).

[6] D. B. Kaplan, M. J. Savage, Nucl. Phys. A556, 653-671 (1993).

[7] N. Kaiser, U. G. Meissner, Nucl. Phys. A489, 671-682 (1988).

[8] N. Kaiser, U. G. Meissner, Nucl. Phys. A499, 699 (1989).

[9] V. M. Dubovik, S. V. Zenkin, Annals Phys. 172, 100-135 (1986).

[10] G. B. Feldman, G. A. Crawford, J. Dubach, B. R. Holstein, Phys. Rev. C43, $863-874$ (1991).

[11] E. M. Henley, W. Y. P. Hwang, L. S. Kisslinger, Phys. Lett. B367, $21-27$ (1996). [nucl-th/9511002].

[12] E. M. Henley, W. Y. P. Hwang, L. S. Kisslinger, Phys. Lett. B440, 449-450 (1998). [nucl-th/9809064].

[13] G. A. Lobov, Phys. Atom. Nucl. 65, 534-538 (2002).

[14] B. Desplanques, J. F. Donoghue, B. R. Holstein, Annals Phys. 124, 449 (1980).

[15] W. Haeberli, B. R. Holstein, In *Haxton, W.C. (ed.), Henley, E.M. (ed.): Symmetries and. [nucl-th/9510062].

[16] W. C. Haxton, [arXiv:0802.2984 [nucl-th]].

[17] M. T. Gericke, R. Alarcon, S. Balascuta, L. Barron-Palos, C. Blessinger, J. D. Bowman, R. D. Carlini, W. Chen et al., Phys. Rev. C83, 015505 (2011).

[18] H. -W. Lin et al. [ Hadron Spectrum Collaboration ], Phys. Rev. D79, 034502 (2009). [arXiv:0810.3588 [hep-lat]].

[19] R. G. Edwards, B. Joo, H. -W. Lin, Phys. Rev. D78, 054501 (2008). [arXiv:0803.3960 [hep-lat]].

[20] S. R. Beane, W. Detmold, T. C. Luu, K. Orginos, A. Parreno, M. J. Savage, A. Torok, A. Walker-Loud, Phys. Rev. D79, 114502 (2009). [arXiv:0903.2990 [hep-lat]].

[21] M. S. Mahbub, W. Kamleh, D. B. Leinweber, A. O Cais, A. G. Williams, Phys. Lett. B693, 351-357 (2010). [arXiv:1007.4871 [hep-lat]].

[22] G. P. Engel et al. [ BGR [Bern-Graz-Regensburg] Collaboration ], Phys. Rev. D82, 034505 (2010). [arXiv:1005.1748 [hep-lat]].

[23] R. G. Edwards, J. J. Dudek, D. G. Richards, S. J. Wallace, [arXiv:1104.5152 [hep-ph]].

[24] J. Dai, M. J. Savage, J. Liu, R. P. Springer, Phys. Lett. B271, $403-409$ (1991).

[25] K. Nakamura et al. [ Particle Data Group Collaboration ], J. Phys. G G37, 075021 (2010).

[26] S. R. Beane, M. J. Savage, Nucl. Phys. B636, 291-304 (2002). [arXiv:hep-lat/0203028 [hep-lat]].

[27] S. R. Beane, W. Detmold, T. CLuu, K. Orginos, A. Parreno, M. J. Savage, A. Torok, A. Walker-Loud, Phys. Rev. D80, 074501 (2009). [arXiv:0905.0466 [hep-lat]].

[28] R. G. Edwards et al. [ SciDAC and LHPC and UKQCD Collaborations ], Nucl. Phys. Proc. Suppl. 140, 832 (2005). [hep-lat/0409003]. 